\title{
Electrodeposition of poly(3,4-ethylenedioxythiophene)/reduced graphene oxide/manganese dioxide for simultaneous detection of uric acid, dopamine and ascorbic acid
}

\begin{abstract}
A simple and sensitive sensor of poly(3,4-ethylenedioxythiophene)/reduced graphene oxide/manganese dioxide modified glassy carbon electrode ( $\mathrm{PrGO} / \mathrm{MnO} 2)$ was fabricated via cyclic voltammetry (CV) for simultaneous detection of uric acid (UA), dopamine (DA) and ascorbic acid (AA). The PrGO/MnO2 composite film possessed excellent electrocatalytic rate and high selectivity towards the oxidation of UA, DA and AA in 0.1 M PBS (pH 6.0). The peak potential separation ( $\triangle \mathrm{Ep}$ ) of AA-DA, AA-UA and DA-UA were 166, 312 and $146 \mathrm{mV}$, respectively. The detection limits of $1.00,0.02$ and $0.05 \mu \mathrm{M}$ with a linear response of $1-800$, 0.03-45 and 0.3-80 $\mu \mathrm{M}$ were obtained for AA, DA and UA, respectively. This sensor also showed an excellent stability (reproducibility and repeatability).
\end{abstract}

Keywords: Reduced graphene oxide; Manganese dioxide; PEDOT; Simultaneous detection; Uric acid; Dopamine and ascorbic acid 